\title{
Qualidade e segurança: caminhos para o sucesso do redesenho do modelo de cuidado obstétrico
}

\author{
Quality and safety: pathways to success of the redesign of the \\ obstetric care model
}

Aspectos relacionados à segurança do paciente vêm se tornando parte fundamental dos processos relacionados à melhoria da qualidade assistencial nos serviços de saúde. O Brasil é um dos países que compõem a Aliança Mundial para a Segurança do Paciente, estabelecida pela Organização Mundial de Saúde (OMS), em 2004, cujo principal propósito é instituir medidas que aumentem a qualidade desses serviços e, por conseguinte, a segurança do paciente. A Agência Nacional de Vigilância Sanitária (ANVISA), a Organização PanAmericana da Saúde (OPAS) e a Secretaria de Assistência à Saúde (SAS), do Ministério da Saúde (MS), vêm trabalhando em conjunto para que esse propósito seja cumprido, contando com a participação de órgãos e entidades da área de saúde, além de instituições de ensino e pesquisa da área de segurança do paciente ${ }^{1-3}$.

Segurança na assistência obstétrica se reveste de grande importância ao considerarmos o elevado número de pacientes envolvidos, uma vez que são aproximadamente 3 milhões de nascimentos acontecendo a cada ano no Brasil, resultando em mais de 6 milhões de pacientes, entre parturientes e recém-nascidos (RNs) ${ }^{4}$. Em 2014, a ANVISA publicou o manual "Serviços de Atenção Materna e Neonatal: Segurança e Qualidade" com o intuito de promover uma atenção obstétrica e neonatal de qualidade, com menos agravos resultantes do próprio processo reprodutivo e/ou relacionados ao processo assistencial, incrementando segurança e humanização ${ }^{5}$.

O conceito mais abrangente de qualidade da assistência em saúde foi introduzido por Avedis Donabedian, na década de 1960. De acordo com esse autor, os sistemas deveriam ser analisados em três áreas: estrutura, processo e resultado ${ }^{6}$. Estrutura inclui todos os fatores que afetam o cenário em que os cuidados são oferecidos, tais como estrutura física, equipamentos e recursos humanos, bem como características organizacionais, como treino e remuneração da equipe. $\mathrm{Na}$ avaliação do processo, as práticas adotadas pelos diversos profissionais são analisadas e julgadas se estão em conformidade com as melhores evidências publicadas, podendo-se expandir essa avaliação para ações tomadas pelos pacientes ou seus familiares. Por meio da análise dos resultados avaliam-se os efeitos da prática no paciente (ou população), incluindo mudanças nas condições de saúde, comportamento ou conhecimento, bem como sua satisfação e qualidade de vida. Com essa proposta, a avaliação da qualidade assistencial é contínua, e não baseada apenas na análise de resultados ou consequências

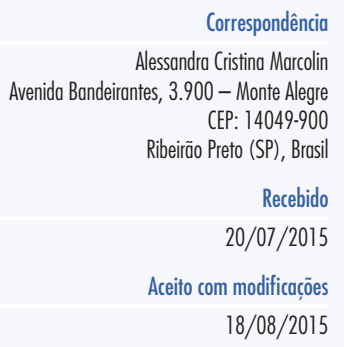

DOI: $10.1590 / 50100-720320150005472$
Departamento de Ginecologia e Obstetrícia da Faculdade de Medicina de Ribeirão Preto da Universidade de São Paulo - USP Ribeirão Preto (SP), Brasil.

'Departamento de Ginecologia e Obstetrícia, Faculdade de Medicina de Ribeirão Preto, Universidade de São Paulo - USP - Ribeirão Preto (SP), Brasil. 
de algum erro ocorrido na prática clínica ${ }^{7}$. Em 2007, o Institute of Healthcare Improvement (IHI) americano adotou a forma de avaliação dos sistemas de saúde proposta por Donabedian. Esse tipo de análise dá subsídios para que as autoridades em saúde possam avaliar o desempenho de sistemas e instituições, a fim de estabelecer um planejamento estratégico que resulte em melhoria na qualidade ${ }^{8}$.

Quando se fala em promoção da qualidade em saúde, inevitavelmente se fala em segurança do paciente e vice-versa. Segurança é o mais importante componente da qualidade e pode ser definida como a prevenção, a melhoria e a correção dos resultados adversos ou das lesões provenientes do processo assistencial, apoiando-se não apenas os pacientes, mas também os profissionais envolvidos. Portanto, para que haja melhora na qualidade dos serviços de atenção materna e neonatal em nosso país, é de capital importância que se proponha um redesenho do modelo de assistência ao parto. Em consonância com esse objetivo, como já citado, o MS e a ANVISA, no final de 2014, anunciaram novas medidas para incentivar o parto normal e reduzir o número de cesáreas (CSs) "desnecessárias" no país, entre outras medidas.

Uma publicação de $2014^{5}$ da ANVISA aborda os passos necessários para a implementação de um programa de promoção da qualidade e segurança na assistência materna e neonatal baseados na proposta do IHI americano ${ }^{8} \mathrm{e}$ adaptados dos trabalhos de Gambone e Reiter ${ }^{9}$, aplicáveis em qualquer local onde se ofereça esse tipo de assistência. Mudanças devem ser promovidas na organização dos serviços, na identificação de prioridades, nas avaliações de desempenho, na identificação de oportunidades para melhorias e nas ações e modificações destinadas a essas melhorias.

Os serviços de saúde variam muito com relação às formas de organização, gestão e ordenamento jurídico. Mesmo dentro do Sistema Único de Saúde (SUS), as instituições podem ser geridas pelos municípios, pelos Estados e pela União, além das instituições universitárias. Ainda, as relações de trabalho entre os funcionários e as organizações variam amplamente, fato que pode incrementar ou comprometer o sucesso de um programa de melhoria da qualidade. Independentemente do tipo de organização do serviço, uma estratégia para garantir a sua adesão a um programa de qualidade, determinada pela Resolução da Diretoria Colegiada (RDC), da ANVISA, n 36 (de 25 de julho de 2013), é a constituição multiprofissional de um Núcleo de Segurança do Paciente, que tem como princípio primordial a melhoria contínua dos processos de cuidado e do uso de tecnologias da saúde ${ }^{3}$.

$\mathrm{Na}$ busca pela melhora da qualidade de um serviço, mudanças não devem ser executadas antes que se definam os objetivos a serem alcançados. Prioridades devem ser identificadas, iniciando-se pela avaliação dos procedimentos mais frequentes e de maior volume, como CS e episiotomias; de situações que apresentam maior risco de complicações, como síndromes hipertensivas gestacionais, hemorragias e prematuridade (espontânea ou eletiva); de eventos-sentinela ou incidentes e de situações relacionadas à organização ${ }^{10,11}$. Os eventos-sentinela demandam ações de notificação, investigação e análise em busca de suas causas, tais como mortes maternas, fetais e neonatais, parto eletivo programado, histerectomia, trauma perineal grave, rotura uterina, transfusão sanguínea, eclâmpsia, abordagem materna ou readmissão hospitalar no puerpério, infecção puerperal, distócia de ombro, complicações anestésicas, admissão materna na unidade de terapia intensiva (UTI) e parada cardiorrespiratória. Como exemplos de problemas organizacionais podem ser citados o elevado tempo de espera para atendimento (eletivo ou de urgência) ou entrega de resultados de exames, prontuário não disponível, defeitos de equipamentos, erro na administração de medicações, violação de protocolo local, conflitos entre membros das equipes no manejo de um caso e parto assistido por pessoa não qualificada.

Qualquer programa para obtenção de melhoria da qualidade dos cuidados maternos e neonatais deve apresentar indicadores ou resultados da eficiência da assistência oferecida. Os indicadores de estrutura, processo e resultado devem se relacionar entre si para que a análise da qualidade seja coerente. Como indicador de estrutura pode-se avaliar se a maternidade possui quartos pré-parto, parto e puerpério imediato (PPP), e como indicador de processo, quantas mulheres estão tendo suas gestações resolvidas nesses locais. Como indicador de resultado pode-se medir o impacto da conduta na redução da taxa de $\mathrm{CS}^{5}$. Alguns indicadores são sugeridos por órgãos internacionais e utilizados em estudos de grande impacto, tais como o Índice de Resultados Adversos, o Escore Ponderado de Resultados Adversos e o Índice de Gravidade ${ }^{11,12}$.

Após a obtenção dos índices de qualidade e a definição das oportunidades de melhorias em um serviço, uma ampla investigação deve ser realizada para se identificar falhas no padrão de cuidados dentro da instituição, com foco no sistema, e não nos indivíduos. Uma metodologia, de caráter retrospectivo, bastante utilizada para esse fim é a análise de causa-raiz (CR), ou seja, que permite a identificação da causa primária ou raiz do problema ${ }^{13}$. Para sua implementação, alguns passos devem ser cumpridos: formação da equipe de investigação, identificação do problema, coleta de informações e evidências, determinação dos eventos relevantes, redução dos riscos e estabelecimento de estratégias de melhorias, implementação de um modelo de redesenho e monitoramento do impacto das novas medidas implantadas.

A equipe de investigação da CR de um evento adverso deve ser multiprofissional e constituída por pessoas 
designadas pelo comitê de segurança local ou pela chefia do serviço. É de grande importância que elas tenham habilidade técnica e emocional para fazer uma análise crítica, imparcial e despretensiosa do evento. Para que haja a identificação do evento, relatórios escritos devem ser solicitados aos envolvidos. Na sequência, procede-se à análise minuciosa do acontecimento, por meio da avaliação de prontuários e de entrevistas com todos os envolvidos. Uma vez finalizada a coleta de dados, a equipe se reúne para discussão das possíveis CRs do evento em questão. A conclusão poderá ser que não ocorreram falhas no padrão de cuidados que levaram àquele desfecho desfavorável e que o resultado foi secundário ao estado do paciente ou a outros fatores não relacionados à assistência. Por outro lado, caso falhas sejam detectadas, a investigação deve ser dirigida aos seus fatores contribuintes, sejam eles causais ou influenciadores, identificando oportunidades para melhoria ${ }^{5}$.

Para o planejamento das ações pode-se utilizar o método PFEA (em inglês PDSA: Plan, Do, Study, Act), ou seja, planeje, faça, estude e aja. O PFEA é um método científico que permite estudo cuidadoso das mudanças a serem implementadas, inicialmente em pequena escala, por meio de tentativa e aprendizado. Para cada mudança, três questões fundamentais devem ser respondidas: $\mathrm{O}$ que estamos tentando atingir (metas/objetivos)? Como saberemos se a mudança é uma melhoria? Que mudanças resultarão em melhorias ${ }^{14}$ ? Sendo assim, cada mudança deverá passar pelas seguintes fases: PLANEJE: os objetivos da intervenção deverão ser estabelecidos, assim como previsões do que poderá acontecer. Um plano deverá ser desenvolvido, definindo-se quem irá executá-lo, de que forma, quando e em quais cenários. Além disso, é preciso que se determine quais dados serão coletados para análise posterior; FAÇA: nesta etapa, com a aplicação da intervenção, os problemas e eventos adversos serão documentados, possibilitando o início da análise; ESTUDE: nesta fase, a análise dos dados será terminada, permitindo o estudo dos resultados e a comparação com as previsões definidas; AJA: neste momento, as intervenções serão modificadas com base na análise realizada e nos novos ciclos de PFEA. Em algumas situações e cenários, vários ciclos serão necessários para que ações que rendam os melhores benefícios sejam definitivamente implementadas.

Felizmente, na assistência obstétrica várias ações já foram testadas e aplicadas, com resultados variáveis em termos de melhorias da qualidade dos serviços prestados, tais como: trabalho em equipe, simulações, adoção de diretrizes baseadas em evidências, desenvolvimento e implantação de protocolos clínicos otimizados, aplicação de listas de checagem de segurança, intervenções combinadas e educação continuada e rondas de segurança ${ }^{5}$. Todas podem ser testadas em ciclos de PFEA, a depender dos objetivos e das prioridades de cada instituição.
Saber trabalhar em equipe é de fundamental importância para a ocorrência de melhorias na qualidade dos cuidados em saúde. Uma equipe deve ter um líder capaz de assegurar esse trabalho em equipe, não por meio do poder ou da intimidação, mas com habilidades de gerenciar os recursos materiais e humanos, encorajar o comportamento da equipe compartilhando informações após um atendimento de emergência ou um resultado adverso e resolver os conflitos, sempre pela perspectiva do paciente ${ }^{11}$. O monitoramento contínuo da prestação de serviços e do ambiente, por cada membro da equipe, também tem relevância, pois pode influenciar positivamente o cuidado do paciente. A existência da cultura do suporte mútuo em uma instituição é outro dado salutar quando almejamos melhorias na qualidade da assistência.

$\mathrm{O}$ treinamento de equipes multiprofissionais em situações de simulação, com manequins ou atores, é de extrema importância para melhorias na assistência obstétrica, uma vez que capacita a equipe no manejo de situações emergenciais sem colocar em risco a vida dos pacientes. O ideal é que essas ações sejam realizadas no próprio local de trabalho, por meio de rápidas aulas teóricas, seguidas por treinamento prático e discussões posteriores sobre o desempenho de cada um. Temas como distócia de ombro, hemorragia puerperal, eclâmpsia, parto vaginal assistido e parto pélvico devem obrigatoriamente fazer parte dessas atividades de simulação em obstetrícia ${ }^{15}$.

A implementação de diretrizes clínicas baseadas em boas evidências deve fazer parte de qualquer programa de promoção da qualidade na assistência à saúde. A ampla variedade de práticas clínicas existentes nos diversos locais de cuidados pode colocar em risco a segurança dos pacientes. Estes podem não estar recebendo o tratamento mais adequado de acordo com as melhores evidências derivadas de estudos científicos bem desenhados, seja pela aplicação inadequada do conhecimento ou pela falta da prática mais eficaz para situações específicas. Exemplos desses protocolos são aqueles publicados pelo American College of Obstetricians and Gynecologists (ACOG) ${ }^{16}$ sobre uso da ocitocina na indução do trabalho de parto, manejo da hemorragia puerperal, da distócia de ombro, da crise hipertensiva na gravidez, da gestação pós-data, do trabalho de parto pré-termo, etc.

Os serviços de atenção materna e neonatal devem implementar listas de checagem de segurança como parte dos seus programas de melhoria da qualidade assistencial. Esses instrumentos são recomendados pelo Institute of Medicine americano desde $1999^{17}$ com o objetivo de prevenir erros durante a assistência. Dentro desse contexto, uma iniciativa interessante foi realizada pela Agência Nacional de Segurança do Paciente (National Patient Safety Agency — NPSA) do Reino Unido, que adaptou a Lista de Verificação de Segurança Cirúrgica da OMS para ser 
utilizada em $\mathrm{CS}^{13}$. Intervenções combinadas (bundles) se originaram dessas listas de checagem de segurança e também devem ser adotadas para garantir qualidade e segurança na assistência. Esses instrumentos existem para garantir que todos os passos de um procedimento sejam realizados toda vez em que for executado ${ }^{18}$. Rondas de segurança semanais nos vários setores da instituição e programas de educação continuada para a equipe multiprofissional de assistência também colaboram para o aumento da qualidade da assistência e para a segurança do paciente.

No Brasil, excelentes iniciativas visando maior qualidade e segurança na assistência materno-infantil têm sido presenciadas, motivadas por programas do MS, publicações e normativas da ANVISA e experiência de outros países. Em 2006, o Institute for Innovation and Improvement, do National Health System (NHS) britânico, por meio da atuação do grupo responsável por garantir qualidade em saúde (Delivering Quality and Value Team), publicou uma série de documentos para ajudar comunidades locais e instituições de saúde a incrementar a qualidade dos cuidados obstétricos, com redução das taxas de CS e aumento dos resultados perinatais normais. O documento intitulado "Focus on: caesarean section" compartilhou com o mundo experiências de serviços britânicos com sucesso nos resultados maternos e perinatais e tem sido uma referência para a comunidade obstétrica brasileira $^{19}$. Essa publicação não debate mais quais são as melhores práticas a serem adotadas, mas como efetuar mudanças necessárias para sua implantação em três esferas principais: manejo da mulher primigesta durante o pré-natal e parto, incremento do parto vaginal após CS e manejo das mulheres com indicação de CS eletiva.

Melhorias na qualidade dos serviços obstétricos também têm sido uma preocupação da saúde suplementar. Em 6 de janeiro de 2015, a diretoria colegiada da Agência Nacional de Saúde Suplementar (ANS) publicou a Resolução Normativa $n^{\circ} 368$, que dispõe sobre o direito de acesso à informação das beneficiárias aos percentuais de cirurgias CSs e de partos normais (por operadora, estabelecimento de saúde e médico) e sobre a utilização do partograma e do cartão da gestante ${ }^{20}$. No mês seguinte, foi finalizado o escopo do projeto brasileiro denominado Parto Adequado, uma iniciativa conjunta da ANS, do Hospital Israelita Albert Einstein e do IHI americano, com o apoio do MS, a ser implementada em hospitais privados e públicos ${ }^{21}$. O objetivo desse projeto-piloto é mudar o modelo de atenção ao parto, por meio de estratégias baseadas nas melhores evidências científicas que aumentem a qualidade e a segurança da assistência nos períodos de pré-parto, parto e pós-parto, incentivando o parto normal e reduzindo CSs "desnecessárias" e possíveis eventos adversos decorrentes de um parto não adequado. Os parceiros da iniciativa reuniram hospitais e operadoras de todo o país para apresentar e discutir essa proposta e, em março de 2015, houve a seleção das instituições participantes: 23 hospitais privados integrantes da rede própria ou credenciada de planos de saúde (1 no Centro-Oeste, 2 no Nordeste, 1 no Norte, 16 no Sudeste e 3 no Sul) e 5 maternidades públicas, com atendimento de partos de risco habitual, que realizam mais de 1.000 partos/ano e com taxas de CS superiores a 60\%. Esse projeto está em fase inicial de implementação, com capacitação dos profissionais envolvidos sobre a metodologia de trabalho e discussão dos objetivos e das estratégias.

Com as informações disponibilizadas até o momento, tornam-se evidentes as preocupações e os esforços das autoridades em saúde, nacionais e internacionais, voltados à obtenção de qualidade e segurança nos cuidados prestados ao binômio materno-infantil. É nesse cenário de muita labuta, de atividades de cooperação multiprofissional, desejo de renovação e elevada expectativa que aguardamos os bons frutos desse árduo trabalho de remodelagem da assistência materna e perinatal.

\section{Referências}

1. Brasil. Ministério da Saúde. Agência Nacional de Vigilância Sanitária. Assistência segura: uma reflexão teórica aplicada à prática. Brasília (DF): ANVISA; 2013.

2. Brasil. Ministério da Saúde. Portaria n. 529 de 01 de abril de 2013. Institui o Programa Nacional de Segurança do Paciente (PNSP). Diário Oficial da União, Brasília (DF) (2013 Abr 2);Sec. 1:43.

3. Brasil. Ministério da Saúde. Agência Nacional de Vigilância Sanitária. Resolução RDC n. 36 de 25 de julho de 2013. Institui ações para a segurança do paciente em serviços de saúde e dá outras providências. Diário Oficial da União, Brasília (DF) (2013 Jul 26);Sec. 1:32.
4. Brasil. Ministério da Saúde. DATASUS [Internet]. Sistema de Informações sobre Nascidos Vivos (SINASC); 2013 [cited 2015 Jul 25]. Available from: <http://tabnet.datasus.gov.br/cgi/tabcgi. exe?sinasc/cnv/nvuf.def>

5. Brasil. Agência Nacional de Vigilância Sanitária. Serviços de atenção materna e neonatal: segurança e qualidade. Brasília (DF): ANVISA; 2014.

6. Donabedian $A$. The quality of care. How can it be assessed? JAMA. 1988;260(12):1743-8.

7. Donabedian A. An introduction to quality assurance in health care. New York: Oxford University Press; 2003. 
8. Martin LA, Nelson EC, Lloyd RC, Nolan TW. Whole system measures. Cambridge: Institute for Healthcare Improvement; 2007.

9. Gambone JC, Reiter RC. Elements of a successful quality improvement and patient safety program in obstetrics and gynecology. Obstet Gynecol Clin North Am. 2008;35(1):129-45.

10. Royal College of Obstetricians and Gynaecologists. Improving patient safety: risk management for maternity and gynaecology. London: RCOG; 2009.

11. American College of Obstetricians and Gynecologists. Quality and safety in women's health care. $2^{\text {nd }}$ ed. Washington (DC): ACOG; 2010.

12. Mann S, Pratt S, Gluck $P$, Nielsen P, Risser D, Greenberg $P$, et al. Assessing quality in obstetrical care: development of standardized measures. Jt Comm J Qual Patient Saf. 2006;32(9):497-505.

13. United Kingdom. National Health System. National Patient Safety Agency. National Reporting and Learning Service [Internet]. Root Cause Analysis (RCA) tools: analysing to identify contributory factors and root causes. Redditch: NRLS; 2010 [cited 2015 May 20]. Available from: <http://www.nrls.npsa.nhs.uk/ resources/?entryid45=75605>

14. Langley GL, Moen R, Nolan KM, Nolan TW, Norman CL, Provost LP. The improvement guide: a practical approach to enhancing organizational performance. $2^{\text {nd }}$ ed. San Francisco: Jossey-Bass; 2009.

15. Dresang LT, Davis S, Miller K, Olden C, Atwood L. Safety in maternity care. In: American Academy of Family Physicians. Advanced Life
Support in Obstetrics (ALSO) Course Syllabus. Leawood: AAFP; 2012. Chapter L, p. 1-15.

16. American College of Obstetricians and Gynecologists [Internet]. [cited 2015 Sept 2]. Available from: <http://www.acog.org/ Resources-And-Publications/Practice-Bulletins-Lisł>

17. National Academy of Science. Institute of Medicine. Committee on Quality of Health Care in America. Crossing the quality chasm: a new health system for the 21 st century. Washington (DC): The National Academy Press; 2001.

18. Minkoff H, Berkowitz R; Greater New York Hospital Association's Perinatal Safety Committee. Fetal monitoring bundle. Obstet Gynecol. 2009;1 14(6):1332-5.

19. United Kingdom. National Health System. Institute for Innovation and Improvement. Delivering quality and value: focus on: caesarean section. Leeds: NHS; 2013.

20. Brasil. Ministério da Saúde. Agência Nacional de Saúde Suplementar. Resolução Normativa - RN n. 368 de 6 de janeiro de 2015. Dispõe sobre o direito de acesso à informação das beneficiárias aos percentuais de cirurgias cesáreas e de partos normais, por operadora, por estabelecimento de saúde e por médico e sobre a utilização do partograma, do cartão da gestante e da carta de informação à gestante no âmbito da saúde suplementar. Diário Oficial da União, Brasília (DF) (2015 Jan 7);Sec. 1:38.

21. Brasil. Ministério da Saúde. Agência Nacional de Saúde Suplementar [Internet]. Projeto parto adequado; 2015 [cited 2015 Ago 3]. Available from: <http://www.ans.gov.br/prestadores/projeto-parto-adequado> 